Du texte à la scène : langages du théâtre

\title{
Le mot et le geste
}

Jean-Michel Déprats

\section{(2) OpenEdition \\ Journals}

Édition électronique

URL : http://journals.openedition.org/shakespeare/473

DOI : 10.4000/shakespeare.473

ISSN : 2271-6424

Éditeur

Société Française Shakespeare

Édition imprimée

Date de publication : 1 novembre 1983

Pagination : 205-224

Référence électronique

Jean-Michel Déprats, "Le mot et le geste », Actes des congrès de la Société française Shakespeare [En ligne], 4 | 1983, mis en ligne le 01 janvier 2007, consulté le 06 mai 2019. URL : http:// journals.openedition.org/shakespeare/473; DOI : 10.4000/shakespeare.473

(c) SFS 
SOCIETE FRANÇAISE SHAKESPEARE

Actes du Congrès 1982

\section{DU TEXTE A LA SCENE : Langages du Théâtre}

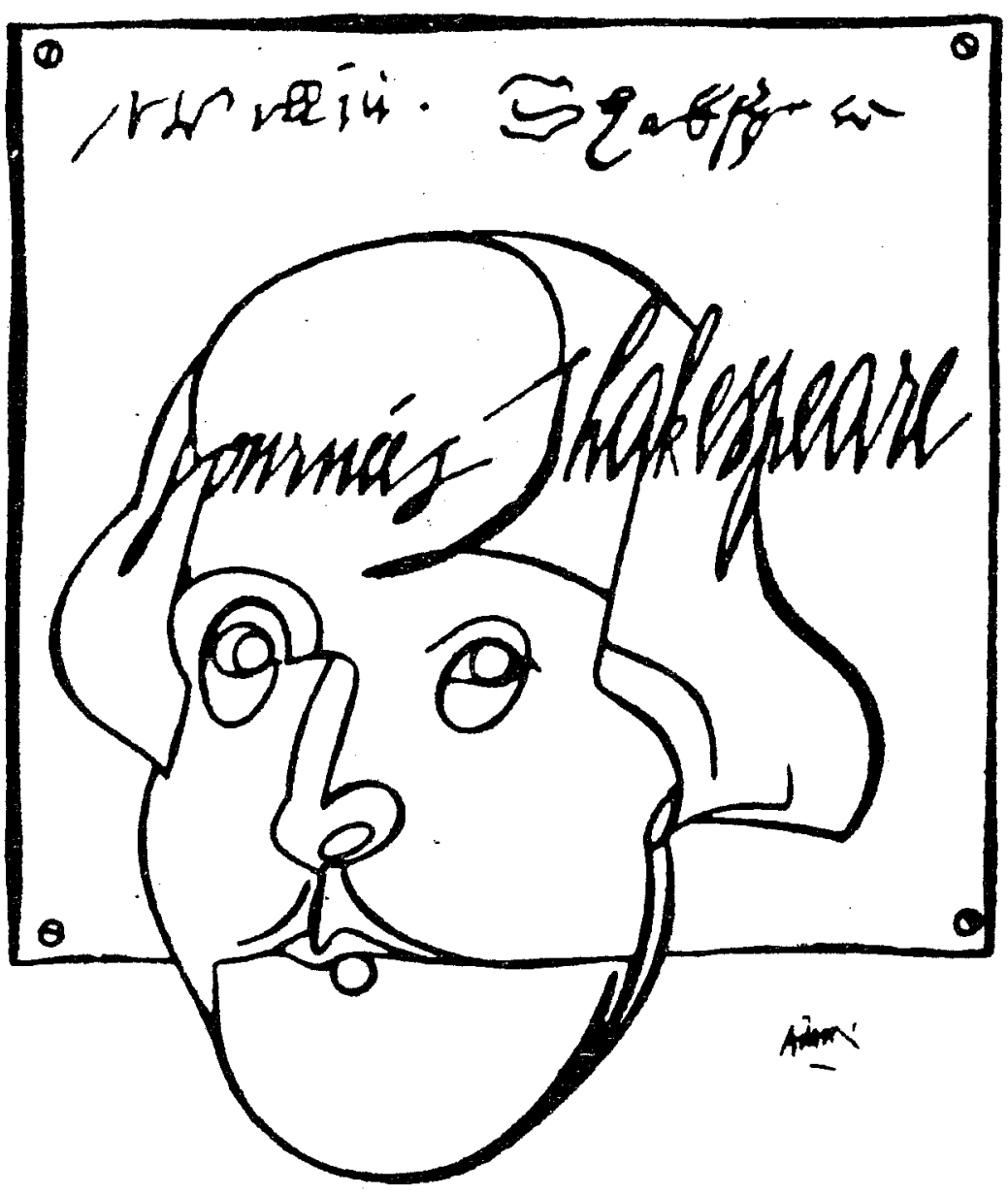

DiRecteur de la publication M.T. Jones - Davies

Publié avec le concours du Centre National de la Recherche Scientifique

JEAN TOUZOT Libraire - Editeur

38 , rue Saint-Sulpice 75278 PARIS CEDEX 061983 
Du Texte à la Scène : Langages du Théâtre. 205

\section{LE MOT ET LE GESTE}

Action is eloquence.

(Coriolanus III.ii.76)

«Do not saw the air too much with your hand, thus, but use all gently; (..) Suit the action to the word, the word to the action.» ${ }^{1}$ Le problème du rapport, de l'ajustement, du mot au geste et du geste au mot, Shakespeare nous le pose déjà par la bouche d'Hamlet.

Je me propose d'explorer quelques uns des rapports du mot et du geste dans le théâtre de Shakespeare : texte et représentation. Ces préliminaires engagés, $j$ 'analyserai dans un deuxième temps les fonctions et l'emploi du geste, la relation de l'image corporelle et de l'image verbale dans deux réalisations récentes : les Peines d'Amour Perdues du Théâtre National de Strasbourg ${ }^{2}$ et le Richard II du Théâtre du Soleil ${ }^{3}$.

Le rapport du mot et du geste ne recoupe pas l'opposition texte/représentation puisque le mot figure dans la représentation sous forme phonique. La première tâche du comédien est en effet de dire le texte, d'être le rhapsode $\mathrm{du}$ poème dramatique. Interviennent dans cette transformation de l'écrit en oral des filtres qui orientent déjà l'interprétation : hauteur, timbre, intensité et volume de la voix, phrasé, accentuation, intonation et rythme de l'élocution. Dans la représentation le mot c'est donc à la fois la couche verbale, le sémantisme, les signes vocaux précédemment énumérés et les propriétés sensibles de l'écriture poétique : rythme, prosodie, tissu métaphorique, musique de la langue. Par geste, j'entendrai non seulement la gectuelle proprement dite : gestes des mains, mouvement des jambes, posture générale par rapport au partenaire et au public mais aussi le mouvement des corps dans l'espace, le déplacement, et le mouvement du visage, 
la mimique. Il y aurait, en effet, quelque artifice à étudier la gestuelle sans son prolongement dynamique, ou à scinder en deux le langage du corps. Mimique, gestuelle et déplacements jouent en général de façon conjointe et le travail du comédien est une totalité.

Il n'y a pas antinomie entre le mot et le geste. Tous deux sont de l'ordre du langage. Dans le discours scénique le geste fonctionne comme un mot dans la phrase. Il peut se traduire par un énoncé : l'agenouillement dénote la prière, la supplication ou la soumission ${ }^{4}$ mais le geste n'est que partiellement lié au langage. Certains gestes ne sont pas verbalisables et presque par définition le geste scénique comporte un élément ludique et une dimension poétique. La longue reptation par laquelle le roi Richard étreint la terre d'Angleterre à son retour d'Irlande (III.ii) dans le Richard II du Théâtre du Soleil est une sorte de poème charnel au sol natal dont le lyrisme excède le sens.

$\mathrm{Si}$ le corps a un langage, le langage aussi a un corps. Pas seulement par métaphore. Il y a un geste de la parole poétique. Dans un texte de théâtre le geste de l'acteur est présent dans la couche verbale sous la forme d'infimes sollicitations musculaires, d'esquisses corporelles. Sans parler des didascalies, le texte dialogué programme, décrit ou suggère des gestes. Tout texte de théâtre appelle son inscription vivante dans le corps, la voix et le jeu de l'acteur. L'ordre des mots, les rythmes, les images sont porteurs de gestes. Pour être compris du spectateur le mot prononcé sur une scène doit être déchiffré par le corps de l'acteur. Il ne suffit pas qu'il soit dit, il faut que tout le corps participe à l'acte de parole. Que de représentations shakespeariennes où le texte paraît ennuyeux, bavard ou surchargé parce que le comédien ne raconte qu'avec sa bouche. Les images du texte sont un instrument de jeu pour le comédien. Ce qui n'implique pas que le texte doit être mimé ou gesticulé; il n'est pire rhétorique théâtrale qu'un mode de jeu où le geste double inutilement la parole. Du mot au geste et du geste au mot il y a un rapport dialectique qui s'enrichit de n'être pas illustratif. 
«Une langue est gestuelle», nous dit Brecht; «lorsqu'elle indique quelles attitudes précises l'homme qui parle adopte envers d'autres hommes» ${ }^{\mathbf{5}}$. Le texte de Shakespeare nous renseigne non seulement sur cette attitude globale du locuteur mais aussi sur son attitude physique et parfois sur celle de l'interlocuteur. Aucun texte de théâtre n'est aussi riche de propositions pour l'acteur quant à la façon dont il doit le prendre en charge, vocalement et corporellement. La parcimonie des indications scéniques est largement compensée par la multitude des indications de mouvement inscrites dans la couche dialoguée. Tantôt de façon explicite : le Fantôme d'Hamlet se déplace d'《un pas digne et raide» : «See, it stalks away ${ }^{6}$. Juliette s'approche $\left\langle\mathrm{d}^{\prime}\right.$ un pied léger ${ }^{7}$ de la cellule de Frère Laurent. Tantôt de façon implicite : à travers les rythmes, les images, la prosodie, Shakespeare donne des suggestions corporelles, oriente la voix et le corps de l'acteur. Antoine exprimant sa fureur après la déroute de sa flotte emploie ce que J.L. Styan appelle une «poésie gestuelle» ${ }^{8}$, c'est-à-dire une poésie qui donne des suggestions corporelles à l'acteur et guide sa voix et son corps :

The shirt of Nessus is upon me : teach me, Alcides, thou mine ancestor, thy rage Let me lodge Lichas on the horns $o^{\prime}$ the moon And with those hands that grasp'd the heaviest

club,

Subdue my worthiest self. ${ }^{9}$

Toutes les propriétés sensibles du vers : les allitérations (chuintantes, sifflantes des deux premiers vers), la courbe mélodique (ascendante dans le troisième vers, descendante dans les deux derniers), la texture auditive (sonorités lourdes des deux derniers vers) traduisent les mouvements $\mathrm{du}$ corps. Antoine se tord de douleur sous l'effet d'une brúlure imaginaire (vers 1 et 2). Il lève les bras au ciel comme pour se libérer de la torture physique et mentale (vers 3 ) et ses bras retombent (vers 4 et 5 ) comme sous le poids de la massue d'Hercule. 
Shakespeare guide l'acteur à travers les images, la prosodie, les rythmes; il imagine ses gestes et ses déplacements. Avec un sens très sûr du mouvement et de l'immobilité sur un plateau de théâtre :

«To fly, to swim, to dive into the fire ${ }^{10}$ se danse tandis que dans cet extrait de Richard II le ritualisme de l'écriture appelle l'immobilité et le hiératisme :

With mine own tears I wash away my balm, With mine own hands I give away my crown, With mine own tongue deny my sacred state, With mine own breath release all duteous

De cette inscription virtuelle du geste dans la physique de la langue on ne peut pourtant pas déduire une mise en scène unique. Les mouvements et les gestes que le texte recèle n'orientent qu'une part de la représentation et la «traduction» scénique des impulsions corporelles doit être abordée sans schématisme. Le texte implique le geste mais ne dicte pas un geste, il ne détermine pas de façon univoque le mouvement du corps et l'inflexion de la voix. Le comédien est libre d'actualiser certaines virtualités gestuelles, d'en laisser d'autres en suspens, libre (et souvent contraint) d'ajouter son propre imaginaire corporel ou de proposer une partition gestuelle en rupture, en «ironie» plutôt qu'en symbiose. La perception des rythmes de l'écriture textuelle n'oblige pas à pratiquer une écriture scénique redondante. Dans la mise en scène de Stuart Seide du Songe d'une nuit d'été ${ }^{12}$, le comédien qui jouait Puck (Jean-Louis Grinfeld) insufflait aux quatre yers que dit Puck avant d'égarer Démétrius et Lysandre dans la forêt $t^{13}$ un autre rythme que celui du texte, un rythme plus lourd, plus insinuant, martelé et haché de silences. La cadence s'accélérait à chaque reprise du premier vers. L'effet produit, celui d'une pulsation tellurique inexorable, d'un bruit sourd qui se propage dans les entrailles de la terre, traduisait l'idée d'ubiquité de Puck avec plus de force que ne l'aurait fait le suivi pointilleux du rythme naturel du texte. 
Le jeu du comédien peut donc entretenir des rapports complexes avec les données textuelles. Dans le théâtre oriental, le geste ne double pas la parole. Plus ou moins proche de la danse, il tient un discours organisé parallèle au discours parlé. C'est le cas du kathakali indien où la symphonie gestuelle travaille en contrepoint du récit chanté par d'autres. Dans le théâtre occidental le geste sert le plus souvent d'accompagnement, d'illustration ou de ponctuation à la parole. Son caractère iconique est fortement marqué, il est puisé dans le code gestuel quotidien, parfois dans un code théâtral construit comme celui de la commedia dell'arte. Mais la rhétorique gestuelle n'est pas nécessairement redondante. On peut faire jouer le geste à l'encontre de ce que disent ou suggèrent les mots. Dans ce cas le geste exprime un non-dit de la parole, un commentaire qui en montre les présupposés ou le mensonge. Lorsqu'il ne fait qu'accompagner ou relayer la parole, il n'a guère besoin d'une lecture autonome : c'est la parole qui lui donne ou lui confirme son sens. Dans les autres cas il a un sens propre et se charge de contenus divers. Analysons cette diversité de contenu en puisant nos exemples essentiellement dans les deux spectacles dont j'ai annoncé une étude détaillée. Le geste peut en premier lieu - c'est le cas le plus fréquent - montrer l'action, indiquer la situation, être un des maillons de la chaîne narrative. Certains moments de cette chaîne narrative, le duel d'Hamlet, la Parade des Preux ou le masque des Moscovites de Peines d'Amour Perdues n'ont d'existence qu'à la représentation. Deuxième fonction du geste : il sert à montrer le personnage dans ses particularités physiques ou psychiques. Le comédien travaille dans une certaine récurrence des mêmes gestes, il choisit une attitude caractéristique, des particularismes gestuels et vocaux. Les trémoussements de plaisir de Jacquinette, la dómarche enfantine de Puce (Moth) sifflotant les mains dans les poches, le maniérisme gestuel d'Armado visent à établir tel ou tel trait distinctif : la sensualité, l'âge, l'affectation. Parfois la gestuelle et la démarche sont 
puisées non dans le vocabulaire gestuel quotidien mais dans un code théâtral ou dans un code culturel au sens large, par exemple cinématographique : Armado marche à grands pas, torse bombé, tête haute comme Matamore, Courge (Costard) a une démarche à la Harpo Marx, contrepoint visuel de ses inventions verbales absurdistes. Dans Richard II, le jardinier et son apprenti ont des gestes, une mimique et une voix de clowns; les bouffons du roi, personnages ajoutés dans la scène du duel judiciaire, rappellent par leurs acrobaties certains personnages du théâtre balinais. La gestuelle de tous les acteurs de Richard II est régie par un code théâtral homogène. De façon significative, il y a peu de traits individualisants. Tous les nobles, peu ou prou, se ressemblent. Le seul exemple d'individualisation n'est qu'une modulation du code. Dans la scène du retour d'exil de Bolingbroke, les comédiens, à la fois cavalier et monture, miment une chevauchée stylisée. L'impulsivité de Percy est connotée par la description gestuelle d'un pur sang qui se cabre sans cesse. C'est une métaphore assez éloquente du caractère du futur Hotspur. Le geste peut aussi montrer le personnage social : ainsi dans Peines d'Amour Perdues la référence iconographique implicite à l'image du roi saint Louis sous son chêne aide à établir la fonction judiciaire du pouvoir royal dans la scène du jugement de Courge (I.i), Le geste enfin sert à dire les passions, à indiquer les sentiments. Dans la mise en scène de Mnouchkine, avec une mimique qui rappelle les films de Kurosawa ou le kabuki, Richard sermonné par Jean de Gand éructe sa fureur. Les yeux exorbités, il semble se dresser sur ses ergots, la joue pâlit de colère comme le suggère le texte. ${ }^{14}$ Telles sont les principales fonctions du signifiant gestuel quand il ne double pas la parole.

Examinons maintenant les rapports du mot et du geste dans la représentation de Peines d'Amour Perdues mise en scène par Jean-Pierre Vincent. La caractéristique majeure de ce spectacle est paradoxalement la clarté. Peines d'Amour Perdues ne passe pas pour une 
pièce facile : si l'intrigue est simple, voire grêlè, la langue est complexe, gorgée de préciosité, de polysémie, d'allusions obscures dans la veine rhétorique de l'euphuisme que Shakespeare pastiche et parodie. Pourtant ce que le spectateur retient surtout ce n'est pas la complexité mais la virevolte et la virtuosité de ce jeu verbal, l'agilité de l'intelligence shakespearienne, la danse des mots en liberté. Cette clarté vient du jeu, de l'orientation donnée au travail des comédiens.

La conviction de Jean-Pierre Vincent est qu'il ne faut pas plaquer, sur un texte de ce genre, un commentaire extérieur. Evoquant ce travail il parle d' exercice athlétique», d'《investissement de vitalité», d'《un phénomène d'immersion dans une réalité imaginaire donnée par un texte», mais déclare qu' "au sens où on emploie ce mot d'habitude il n'y a pas de mise en scène dans ce spectacle ${ }^{15}$. Contre la prolifération des signes visuels dans un certain thêâtre contemporain, il revendique un théâtre de l'écoute. Pas de lecture personnelle ou de volontarisme signifiant mais un désir de transparence, une volonté de coller de façon rigoureuse au surgissement des images et à l'économie rhétorique du texte. De fait il n'y a qu'un tout petit nombre de moments ou de microsituations où l'on peut voir affleurer une lecture construite : l'arrivée de Mercade et la minute de silence qui par une rupture violente instaure une mise en suspens de la représentation et une cassure de l'univers de la comédie $^{16}$, l'effondrement d'Armado à l'avant-scène quand le masque tombe et que se révèle la vérité intérieure du personnage ${ }^{17}$. Partout ailleurs la réalité scénique semble sourdre des mots et des images du texte. J.-P Vincent pense qu'on peut déduire de la composition énergétique du texte et de la durée présumée de la représentation un certain nombre de principes de jeu. La nécessité tout d'abord d'un jeu rapide, d'un flux continu d'énergie et d'une élasticité vocale, physique et émotionnelle constante chez le comédien. Ce n'est pas en ralentissant mais en accélérant qu'on fait comprendre ce 
type de texte ${ }^{18}$. Encore faut-il que le comédien soit porteur de toute la vibration et la charge sensible des mots, que son jeu soit nourri par une compréhension imaginative corporelle et mentale du texte. Comment y parvient-il? En faisant travailler en lui un système d'images cohérent avec la chaîne des images du texte ${ }^{19}$

Le travail du comédien est un travail de connivence athlétique et imaginative, d'identification physique et mentale avec la chaîne des mots qu'il est en train de dire. Alan Howard disait dans une interview que si on le tuait et si on le disséquait au moment où il était en train de proférer une image sur scène, l'autopsie montrerait que l'ensemble de son corps était cette image. Dans Peines d'Ainour Perdues, lorsque l'image est simple, le geste que suscite la mise en mouvement du corps peut etre redondant. Ainsi Berowne semble englué dans une boue imaginaire quand il dit : «Je me piège à la glu, la glu qui poisse, poisse, le sale mot!» ${ }^{20}$ Tancé par Rosaline il mime les épreuves qu'il veut se voir infliger :

Me voici devant toi, ma dame; braque ta malice contre moi

Blesse-moi de ton mépris, accable-moi de tes sarcasmes,

De ton esprit pointu perce mon ignorance, Hache-moi en morceaux du tranchant de ta pensée. ${ }^{21}$

A d'autres moments, la chaîne des mots est plus métaphorique ou plus complexe, le jeu n'est pas perçu comme redondant parce que l'acteur fait travailler en lui un réseau d'images plus autonome par rapport aux mots du texte. Car il ne s'agit pas de mimer le texte mais de l'incorporer. La capture de l'énergie mentale ou sonore d'un mot n'appelle que rarement une traduction gestuelle illustrative. Les seuls personnages qui miment le texte sont les pédants. Dans la gestuelle d'Holopherne, la manie du synonyme a pour corollaire une mimique et une gesticulation pléonastiques. "Imitari n'est rien», dit-il, joignant le geste à la parole :«c'est ce que fait le lévrier avec son 
maître, le singe avec son gardien, le cheval bien dressé avec son cavalier $\rangle^{22}$. Lorsqu'Armado décrit les effets du rire qui «dilate la rate» et «gonfle les poumons», la glose gestuelle double l'exégèse verbale déjà redondante ${ }^{23}$. En revanche, de nombreux passages qui pourraient être mimés sont portés par une pure acrobatie verbale. C'est le cas de la scène de la rémunération ${ }^{24}$ qui $a$, avec la reprise $\mathrm{du}$ spectacle, subi une évolution significative. Dans la première version créée en Avignon, le comédien jonglait avec la pièce de trois liards. Le jeu verbal s'appuyait sur le jeu scénique. Dans la nouvelle version, il met la pièce à la poche tout de suite. Le commentaire est purement verbal. Cette évolution qui tend à aller vers une économie théâtrale plus grande, vers une purification du geste a marqué tout le travail. Par exemple celui de la scène de l'envoi et de l'oie qui a suscité tant de perplexité et de glose. Au début des répétitions les comédiens avaient le sentiment de se trouver devant un rébus indéchiffrable, jusqu'au jour où ils ont découvert que le texte tissait une série de jeux de mots et de sous-entendus obscènes. Il y a, en effet, une trame verbale qui relie les références génitales - branle français (French brawl) quilles coupées (broken shin) - aux références anales - envoi/clystère/ purgatif/lâcher (envoy/enema/purgation/let me loose) en passant par les références à la prostituée - oie (goose) affranchie (one Frances) ${ }^{25}$ - Dans un premier temps les comédiens mimaient tout. Au fil des répétitions la rhétorique gestuelle se sclérosait et l'effet comique commençait à se tarir. Tous les gestes ont été coupés mais les comédiens étaient tellement nourris de sous-entendus que le texte seul s'est mis à évoquer tout naturellement une poétique obscène du bas-ventre.

Il y a en revanche des passages où la gestuelle et les déplacements ont un rôle mimétique du fonctionnem:nt formel du texte. Ainsi les chassés-croisés de la Princesse, Maria, Catherine, Boyet, à la fin de l'acte II, spatialisent le jeu de répliques lancées conme des balles qui s'entrecroisent autour de quelques vocables répétés 
(mot et bon mot, béliers, pâture ${ }^{26}$ ). Ailleurs Rosaline et Boyet se déplacent le long d'une circonférence de façon diamétralement opposée, s'épient à distance respectable et décochent leurs traits d'esprit jusqu'à ce que l'un des deux fasse mouche ${ }^{27}$. Ce mode d'échange verbal plus acerbe est métaphorisé dans l'espace à travers la figure du cercle.

Dans certains cas c'est une structure syntaxique que traduit la gestuelle : ainsi le comédien (Jean-Louis Fayollet) souligne de gestes de la main et du bras la rhétorique guindee de la gradation : adjectif, comparatif, superlatif, dans la déclaration d'amour d'Armado : "J'aime jusqu'au sol - vil - que son soulier - plus vil - guidé par son pied - très vil - foule $\gg^{28}$.

Il est intéressant de se demander également sur quel type de décalage entre le mot et le geste - ou entre deux gestes - s'appuient les effets comiques. La main levée en signe de fureur et le regard menaçant de Berowne ponctuant l'insulte suffisent à faire chuter Courge $^{29}$. Ce comique joue sur l'anticipation, sur le court-circuit entre l'intentionnalité du mot et le geste. On peut dire aussi que le mot est doté de l'efficacité du geste. De nombreux jeux de scène s'appuient sur une méprise verbale. Le comique consiste à prendre au pied de la lettre le mot qui de toute évidence est employé au sens figuré ou a valeur de métaphore. L'auditoire d'Armado scrute dans le ciel les retombées des «explosions d'allégresse» ${ }^{30}$ que celui-ci évoque, ou suit des yeux le passage imaginaire du Matamore et d'Holopherne à chaque "passons» ${ }^{31}$ dont Armado ponctue son discours. L'invention du gag gestuel consiste donc à rechercher ce qui permet au spectateur, par un court-circuit du processus de métaphorisation, de comprendre le mot dans son sens littéral. «Monsieur, vous avez renversé Alisandre le Grand $\rangle^{32}$, dit Courge à Nathaniel qui se parvient pas à se relever. La prestation est renversante en effet, mais c'est plus sous le poids d'une lance de bois que par la qualité de son interprétation. Dans d'autres cas, le geste joue à l'encontre de ce que dit 
Du Texte à la Scène :Langages du Théâtre.

le mot. Ainsi Armado s'essouffle à évoquer dans une seule respiration :

... L'héritier d'llion

Qui avait un tel souffle qu'il pouvait lutter

Du matin jusqu'au soir hors de son pavillon. ${ }^{3} 3$

Le plus souvent l'effet comique joue donc sur un décalage entre le mot et le geste, sur un brouillage du processus de signification.

Tous les gestes analysés jusqu'à présent opèrent dans une relative transparence, ils sont aisément déchiffrables. Il arrive aussi que le geste soit opaque, que son fonctionnement relève d'une communication induite, de corps à corps, non verbalisable. Jacquinette demandant au curé de lui lire la lettre qu'elle a reçue d'Armado est prise d'un léger sautillement qui se communique à Messire Nathaniel. $\mathrm{Ce}$ dernier finit par trébucher comme pris de vertige. Ce geste appartient essentiellement à un registre ludique, au plaisir de la gesticulation gratuite et ne gagnerait rien à être décrypté.

Cette mise en scène de Peines d'Amour Perdues se caractérise donc par une conjonction de l'image corporelle et de l'image verbale. La gestuelle apparaît en continuité avec la parole, elle affleure à partir d'elle. Même si par endroits le geste semble mimer le texte, il est surtout l'indice d'une incorporation du texte et n'est pas donné à lire de façon autonome. Ce qui est recherché c'est une organicité du corps et du verbe, une totalité de sons et de gestes. Cette symbiose du mot et du geste implique une certaine transparence de la représentation et une forme de mimesis qui s'oppose nettement à la théâtralité exacerbée du Théâtre du Soleil.

Ariane Mnouchkine construit son Richard II sur une forme. Au thêâtre oriental, notamment japonais, elle emprunte non un code ou un style de jeu mais un imaginaire et une écriture scénique qui donnent une forme aux passions et un dessin précis dans l'espace aux états des personnages. La référence japonaise a souvent été perçue comme une référence de contenu ${ }^{34}$, alors qu'il s'agit 
essentiellement d'une référence théâtrale.

A. Mnouchkine ne conçoit pas la chimie de la transformation d'un texte en corps et en gestes sans la médiation d'une forme : forme clownesque dans Les Clowns, théâtre forain et marionnettes dans 1789 , commedia dell'arte dans L'Age d'Or, kabuki et théâtre balinais aujourd'hui dans Richard II, kathakali et barata natyam dans La Nuit des Rois. A. Mnouchkine travaille sur des formes théâtrales pures parce que ces formes permettent d'échapper à la contrainte du réalisme esthétique ${ }^{35}$. Pour Richard II les formes du théâtre japonais ont été choisies parce que ce sont des formes royales capables d'exprimer un univers rituel et chevaleresque. Il ne s'agit toutefois ni de reconstitution ni de recréation mais d'une influence. Les entrées caracolantes, les chevauchées stylisées, les postures d'immobilité sauvage, jambes en équerre face au public, constituent un rituel inventé qui a pour effet premier d'annuler tout psychologisme et d'instaurer une distance. $\mathrm{Ce}$ rituel est composite. Il y a croisement entre les signes $\mathrm{du}$ corps des acteurs orientaux et les gestes des acteurs occidentaux. La mimique de Richard emprunte autant à la mimique de Pierrot qu'à l'expression des passions dans le théâtre kabuki.

$\mathrm{Du}$ point de vue qui nous intéresse ici, le trait le plus marquant est la dissociation du geste et du mot. Le geste ne suit pas le texte, il ne l'illustre pas, ne le traduit pas. Il est rare même qu'il le métaphorise car il appartient à un code théâtral autonome dont la fonction est de dire, autre chose que le texte. La position de base dans les scènes de cour : jambes arquées, mains sur les cuisses, le corps animé d'un ample respiration, est une transposition de l'homme divinisé, de l'homme-Dieu. Le Roi et les Grands du royaume forment une sorte d'Olympe avec un Richard II Jupiter, un York Cronos, etc. ${ }^{36}$. Cette gestuelle joue sur un nombre restreint de postures. A certains moments les comédiens se figent pour dire le texte comme si le travail d'interprétation passait alors essentiellement par la diction. Naturellement, une posture statique ne demande pas moins 
de jeu, d'investissement et de concentration que des évolutions dynamiques mais l'émission vocale se fait le plus souvent dans une attitude statique. Dans cette posture les comédiens deviennent les conteurs du texte, les récitants du poème dramatique ${ }^{37}$. La fonction instrumentale l'emporte sur la fonction de jeu. Les mots sont lancés vers le public, surarticulés, proférés avec une tension de l'élocution qui rappelle la diction de Bruce Myers dans Timon. Ce n'est pas une déclamation ou une récitation mais une profération dont le caractère de stylisation est fortement marqué. Cette profération ne suit pas le phrasé naturel. Le texte est dit recto tono, chaque syllabe a la même durée et les syllables élidées sont prononcées avec la même force que les autres. La voix est soutenue par un accompagnement rythmique de tambours thailandais, de cloches et de gongs tibétains, de tablas mais aussi d'instruments à vent ou à cordes. Le découpage syllabique conduit à une écoute plus aiguë des données matérielles du texte en particulier des allitérations, sensibles par exemple dans ces paroles de Jean de Gand à Bolingbroke :

Que tes coups doublement redoublés

S'abattent comme un stupéfiant tonnerre

Sur le casque de ton adverse et perfide ennemi! ${ }^{38}$

Il y a un plaisir de la restitution sonore des structures matérielles de la langue. Mais au bout d'un certain temps la raideur prosodique devient monotone et sature l'écoute. Elle étire le temps de la représentation déjà distendue par la longueur de la version française ${ }^{39}$.

Pourtant cette interprétation du texte procède d'une intuition à mes yeux forte et juste sur la nature du texte shakespearien. A l'exception notamment des réalisations de Peter Brook, de nombreuses mises en scène skakespeariennes, conditionnées par les données spatiales du thêâtre à l'italienne, restent tributaires du réalisme psychologique. Au lieu de projeter le texte vers le public dans une communication directe avec le spectateur, les comédiens se parlent entre eux. Avec l'adresse directe au public $^{40}$, A. Mnouchkine retrouve une des dimensions 
essentielles du jeu shakespearien ${ }^{41}$. Le mode de diction adopté annule tout psychologisme et donne au texte un statut de transcendance par rapport aux personnages. I1 produit une sorte de ritualisation et de désindividualisation de la parole poétique. Sans porter atteinte à la différenciation des comportements verbaux il permet d'entendre aussi le rebond d'une image d'un rôle à l'autre; il crée un continuum verbal, un espace de parole homogène et le texte parle autant que les personnages le parlent. Il n'est pas seulement la voix de leur intériorité, il est aussi la voix de l'histoire en train de se faire, la voix du monde en train de se dire. S'agissant d'un texte dans lequel la formalisation et la ritualisation de l'écriture sont aussi marquées, on ne récusera pas, a priori, un mode d'interprétation qui restitue cette dimension textuelle.

Un exemple précis donnera une idée exacte du traitement du texte et des rapports de la parole et de la gestuelle. La scène ii de l'acte I se termine par une poignante confession de la duchesse de Gloucester qui, après avoir exhorté Jean de Gand à la vengeance, essaie de le retenir. Elle a dit tout ce qu'elle avait à lui dire. Pourtant la détresse et la solitude demeurent. Elle s'exprime soudain de façon directe dans un texte haletant, haché de silences et d'hésitations:

Un mot encore. [...]

Je prends congé de vous avant d'avoir rien dit

Car le chagrin ne finit pas quand il semble épuisé.

Recommande-moi à ton frère York.

Voilà ... c'est tout ... Non mais ne t'en va pas

ainsi.

Bien que ce soit là tout, ne t'en va pas si vite.

Je vais me rappeler autre chose ...

Dis lui ... Ah! quoi donc ?... Dis lui

De venir me voir au plus vite chez moi. 42

$\mathrm{Si}$ on se laissait guider par les sollicitations musculaires et émotionnelles du texte, par les silences et les hésitations, cette écriture du désarroi, relayée par une gestuelle et des déplacements naturalistes, aboutirait à une 
mobilité fiévreuse faite d'impulsions retenues, de mouvements avortés, d'une errance accablée et sans but. Dans la mise en scène de Mnouchkine les deux interlocuteurs sont immobiles, assis à distance respectable, face au public. La comédienne n'indique pas par des différences de phrasé, de rythme, d'intonation, de position de la voix, les divers mouvements psychologiques. Elle interprète le texte de façon globale. La tirade est dite d'une voix chaude, vibrante, mais sans rupture de ton et sans hésitation. C'est une sorte de lamento funèbre ininterrompu dans lequel l'expression de la douleur est extrêmement formalisée. La voix seule et ses infimes modulations sont chargées de dire le sens et l'émotion.

Souvent, parce qu'elles jouent par contraste, la fixité de la gestuelle et la posture statique ont une grande force narrative. Après la mort de Gand et la saisie des biens de Bolingbroke, Northumberland, Ross et Willoughby restent seuls sur scène ${ }^{43}$. C'est une scène de complot faite d'observation et de méfiance réciproques, une scène où le discours s'avance masqué. Au lieu de jouer sur des déplacements circulaires, sur des silences, sur des attaques en oblique, les trois comédiens sont là encore face au public, les mains sur les hanches et parlent sans se regarder. Le complot et la difficile émergence de la parole n'en ressortent que mieux. Mais il y a d'autres modes de rapport entre le mot et le geste. A la gestuelle du personnage, aux gestes du rituel théâtral: postures d'homme-Dieu, entrées rythmées, courses-poursuites, il faut ajouter naturellement une gestuelle illustrative, celle qui accomplit les actes programmés par le texte. «Voici mon gage», «le Roi a jeté son bâton», «Ma nièce, venez avec moi» sont des énoncés qui exigent évidemment le geste qu'ils décrivent. Il y a les gestes à valeur illocutọire ${ }^{4}$ : Mowbray déclarant «Si je suis un traître, une fois sur mon cheval, puissé-je tomber mort $\rangle^{45}$ concrétise son voeu par une chute volontaire. Il y a les gestes qui traduisent une réaction psychique. Condamné à l'exil sans espoir de retour, le même Mowbray titube comme s'il ployait sous le poids d'un 
fardeau imaginaire. L'élément marquant de cette gestuelle est que le geste est stylisé, formalisé; il tend vers la danse. Bolingbroke banni quitte la terre d'Angleterre à regret. Il s'avance, hésite, est poussé par son père, s'avance, hésite, est poussé par son père. Ce rythme ternaire répété à une vitesse croissante devient une chorégraphie gestuelle, une danse stylisée que cadencent les percussions. Cet élément de répétition et d'amplification poétique ${ }^{46}$ se retrouve dans la scène où Richard se dépose lui-même et se dépouille lentement de ses vêtements royaux dans une sorte de temps éternisé (IV, i). Là geste et texte jouent en contrepoint car la métaphore vestimentaire est explicitement donnée par le texte ${ }^{4}$. Une autre scène métaphorise une situation par élargissement dans l'espace : celle où Richard enchaîné est emmené à la Tour $(V, i)$. Dans le spectacle du Théâtre du Soleil, Richard est comme une araignée au milieu de sa toile attaché par d'immenses chaînes que tiennent en fond de scène des gardes vêtus de noir. Il y a enfin l'utilisation plastique du geste pour créer un tableau ou un emblème comme dans la belle image de la Pietà qui clôt le spectacle ${ }^{48}$.

L'inscription du geste dans le discours scénique se caractérise donc par la mise en oeuvre d'une gestuelle non naturaliste, stylisée, à laquelle est dévolue le plus souvent un discours autonome. Le geste ne s'efface pas derrière le mot, il ne l'illustre pas; on pourra estimer que l'image scénique ou vocale bloque parfois l'imaginaire du texte. Pourtant l'adoption d'une diction qui privilégie l'écoute marque certainement la volonté de laisser les mots de Shakespeare s'envoler librement vers le spectateur. Le choix d'un espace de jeu libre témoigne aussi du désir de faire de l'acteur-nu-disant-les-mots le centre de la représentation.

Ce rapport direct au texte, les deux spectacles tentent donc d'y parvenir par des procédures différentes : dans Richard II, disjonction de la gestuelle et de la parole, dans Peines d'Amour Perdues, fusion du geste et du son. $\mathrm{Ce}$ ne sont là pourtant que quelques uns des modes d'arti- 
culation du mot et du geste dans le langage thêatral. Il y a une multitude de rapports envisageables mais ce qu'il convient de ne pas oublier, c'est que même s'il ne joue pas de façon autonome, le geste est un langage qui recèle des possibilités d'expression et de signification immenses. Le théâtre n'a rien à gagner d'une sclérose du vocabulaire gestuel, d'un geste analphabète ou balbutiant. Quand le corps est muet, la parole est morte. Au théâtre le verbe est chair - la parole est lourde de toute la matérialité d'un corps. Ce corps, comme le rappelle Ulysse décrivant Cressida, parle :

There's language in her eye, her cheek, her lip, Nay, her foot speaks; her wanton spirits look

out

At every joint and motive of her body. ${ }^{49}$ 


\section{NOTES}

1. Hamlet, IIl .ii. 5-7;17-18.

2. Créé le 2 février 1982 au Thé âtre National de Strasbourg.

3. Créé le 10 décembre 1981 à la Cartoucherie de Vincennes.

4. Encore que par son inscription contextuelle ce même geste puisse acquérir une résonance symbolique qui en dépasse le sens habituel : cf. l'agenouillement de Volumnie devant Coriolan (Corlolanus Viii.56-59), celui de Lear devant Cordelia (King Lear IV.vii.59) ou celui de lago et d'Othello qui scelle le pacte diabolique de leur alliance (Othello III iii.462-9)

5. Ecrits sur le Théâtre, L'Arche, t. 1, p. 462 .

6. Hamlet I. i. 50.

7. Romeo and Juliet, II.vi.16.

8. J.L. STYAN, Shakespeare's Stagecraft, CUP, 1967 , pp. 53 et 56 . C'est à lui que j'emprunte les exemples cités ici.

9. Antony and Cleopatra, IV.xii.43-47.

10. The Tempest I.ii.190-191.

11. R ichard II IV i.207-210.

12. Théâtre National de Chaillot, janvier 1982 .

13. A Midsummer Night's Dream, III.ii.396-400.

14. «Im bécile lu natique à la maigre cervelle, A busant du privil ège de la fièvre!

Tu oses faire pâlir notre joue par ton sermon glaçant Qui de fureur a chassé le sang royal loin de sa résidence natu relle»

Richard II, II.i.115-118. Trad. Ariane Mnouchkine, Solin, Paris, 1982, p. 39 .

15. «Un théâtre de l'écoute», entretien avec J.P. Vincent, Théâtre/Public, N 46/47,pp. 19-21.

16. «Sous les coups de boutoir de ces femmes venues de loin, les illusions vont se dissoudre. Et le théâtre avec. Enflé, dédoublé jusqu'à devenir théâtre dans le théâtre, illusion dans l'illusion, le théâtre lui-même bute contre la péripétie finale et comme il est dit sur la scène même la comédie ne finit pas comme les vieilles pièces» J.P. VINCENT, «Le Chemin des Ecoliers», T.N.S. A ctualités, $\mathrm{N}^{0} ! 45, \mathrm{p} .6$.

17. Cf. J.M. DEPRATS, «Armado»,Ibid., p. 12. 
18. Cf. Rencontre avec J.-P. Vincent - Société française Shakespeare. Actes du Congrès 1980 , Jean Touzot, éd., p. 151 .

19. Cf. J.-P. VINCENT, «Un théâtre de l'écoute», loc. cit. pp. $19-20$.

20. Peines d'Amour Perdues (IV iii. 2-4). Trad. J.M. Déprats, Presses de l'IREG, Strasbourg, 1982, p. 59.

21. Ibid., p. 93 (V. ii. 396-399). C'est nous qui soulignons les mots qui font l'objet d'un jeu illustratif.

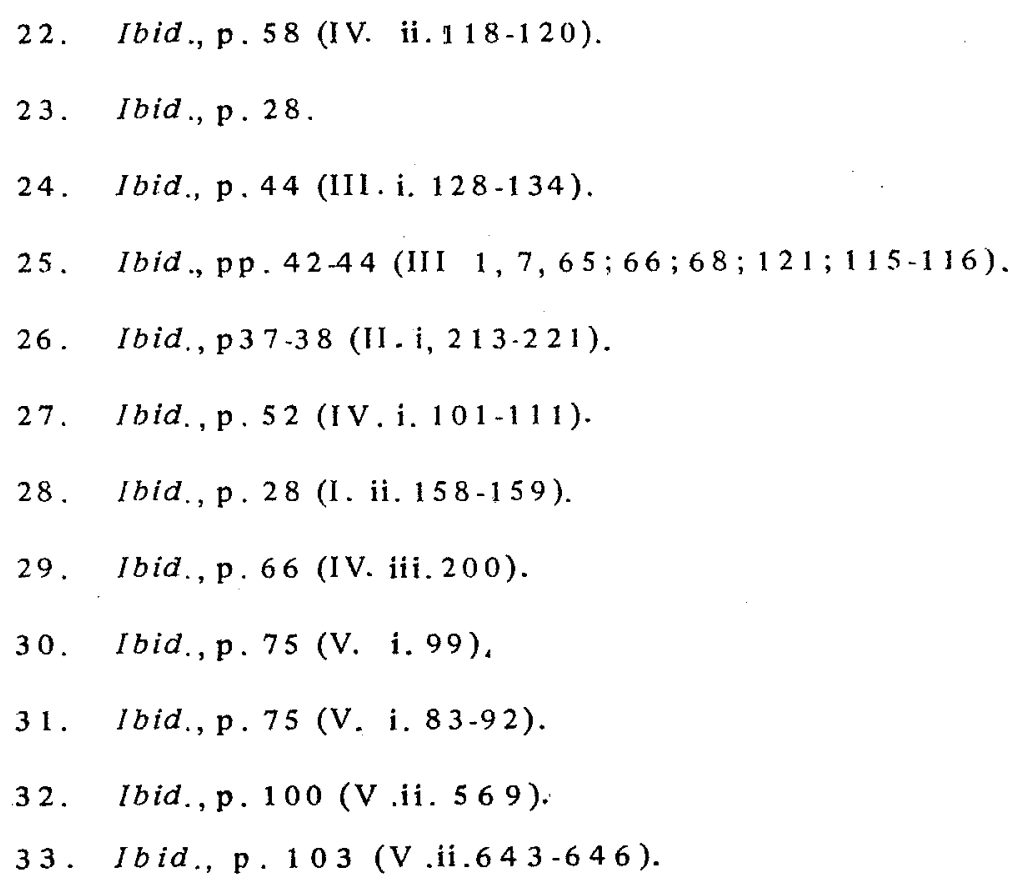

34. Certes ce croisement entre les shogun et la dynastie des Plantagenets s'explique aussi par le besoin de donner des images fortes de la féodalité. Et si les combats des fauves féodaux de l'Angleterre de la fin du Moyen Age sont déchiffés à la lumière d'un autre Moyen Age, l'image est ravivée mais elle n'est pas faussée. Ariane Mnouchkine estime que les images de la féodalité sont plus vivantes pour nous dans certains films japonais ou dans certains romans japonais que dans nos représentations du Moyen Age, de Thierry la Fronde aux gravures des $R$ iches Heures du duc de Berry $C f$. "Le besoin d'une forme \%, Entretien avec A. Mnouchkine, Théâtre/Public, , No 46/47, pp. 8-11.

35. Cf. Entretien avec A. Mnouchkine, Théâtre/Public, No $5 / 6$, p. 5 .

36. $C f$. «Un texte masqué», Entretien avec Georges Bigot et Philippe Hottier,Théatre/Public, No 46/47,p. 13.

37. Sur ce point également il y a continuité dans le travail du Théatre du Soleil. Dans 1789 les comédiens étaient avant tout 
les récitants de la fable. Ils prenaient en charge chacun une partie du public pour lui raconter la prise de la Bastille. Dans L'Age d'Or, Philippe Caubère/Abdallah avait aussi cette fonction d'énonciation de la fable.

38. Richard II. Ibid., p. 20 (1.iii.80-82).

39. Il n'est pas rare que les dix pieds du pentamètre soient traduits par des «vers» de quinze, dix-sept, voire dix-neuf syllabes (ex. I. i. 81 , trad. op. cit., p. 10). Malgré de nombreuses coupures, la représentation dure $4 \mathrm{~h} 30$.

40. Cf. Ariane MNOUCHKINE : Chaque fois qu'en répétition les comédiens se retrouvaient en train de se parler, ça ne marchait pas. Je leur disais : «Racontez au public. Le texte de Shakespeare est fait pour etre dit comme ça. Dès qu'on commence à moduler, à raffiner, à subtiliser, on édulcore». Entretien avec A. Mnouchkine, op. cit., p 8 .

, 41. Cf. J.L. STYAN : «It is probable that Shakespeare saw his actors as playing to their audience most of the time», op. cit., p. 75 .

42. Richard II (I. ii. 58-66) op. cit., p. 17.

43. On pourrait faire la même remarque pour d'autres séquences où l'on retrouve la $m$ ême disposition : scène du commen. taire des adieux de Bolingbroke entre Richard, Aumerle, Bushy et Bagot (I.iv), scène où Bushy et Bagot tentent d'apaiser le chagrin de la Reine (II ii ).

44. La théorie austinienne des actes de langage distingue le locutoire (communication du contenu factuel du discours), 1 e perlocutoire (action émotionnelle sur le destinataire) et l'illocutoire (action réelle de la parole. Cf. J.L. AUSTIN, Quand dire, c'est faire, Seuil, 1970, p. 219 sq.

45. Richard II (I. i. 82) op. cit., p. 10.

46. Caractéristique d'une esthétique dont le film Molière offre de nombreux exemples: la scène du carnaval, des gondoles, du tréteau poussé par le vent et la séquence de la mort de Molière.

47. «J'ai de mon âme donné consentement/Pour dépouiller le fastueux corps d'un Roi» (IV.i. 250) Richard II, op. cit. p. 103.

48. Sur cette image de la Pietà, voir Théâtre/Public, No $46 / 47$, p. 14 .

49. Troilus and Cressida IV.v.55-5 7. 